\title{
TEOR E CAPACIDADE MÁXIMA DE ADSORÇÃO DE ARSÊNIO EM LATOSSOLOS BRASILEIROS ${ }^{(1)}$
}

\author{
Mari Lucia Campos ${ }^{(2)}$, Luiz Roberto Guimarães Guilherme ${ }^{(3)}$, Rafael \\ Siqueira Lopes ${ }^{(4)}$, Alessandra Silveira Antunes ${ }^{(5)}$, João José Granate \\ de Sá e Melo Marques ${ }^{(3)} \&$ Nilton Curi ${ }^{(3)}$
}

\begin{abstract}
RESUMO
A alta toxicidade do As aos animais e humanos e a possibilidade de existência de grande número de áreas contaminadas tornam imprescindível o conhecimento do teor semitotal em solos ditos não-contaminados e dos processos de adsorção do As em solos de carga variável. O objetivo deste trabalho foi determinar o teor e a capacidade máxima de adsorção de As $\left(\mathrm{CMADS}_{\mathrm{AS}}\right)$ em Latossolos. $O$ teor total foi determinado pelo método USEPA 3051A, e a $\mathrm{CMADS}_{\mathrm{As}}$, com auxílio de isotermas de Langmuir com base nos valores de adsorção obtidos em dose de As (0, 90, 190, 380,760 e $1.150 \mu \mathrm{mol} \mathrm{L}^{-1}$ ) (relação solo:solução final = 1:100), a pH 5,5 e força iônica de $15 \mathrm{mmol} \mathrm{L}^{-1}$. Os 17 Latossolos apresentaram teor médio total de As de $5,92 \mathrm{mg} \mathrm{kg}^{-1} \mathrm{e} \mathrm{CMADS}_{\mathrm{As}}$ média de $2.013 \mathrm{mg} \mathrm{kg}^{-1}$. O teor de argila e os óxidos de Fe e $\mathrm{Al}$ apresentaram influência positiva na $\mathrm{CMADS}_{\mathrm{As}}$.

Termos de indexação: sorção, contaminação química, poluente, elementos-traço.

\section{SUMMARY: CONTENT AND MAXIMUM CAPACITY OF ARSENIC ADSORPTION IN BRAZILIAN OXISOLS}

In view of the toxicity of As for man and animals and the possibility of existence of a great number of contaminated areas it is imperative to know the total As content in soils considered uncontaminated and about As sorption processes in soils of variable charge. The objective of this work was to determine the total content and maximum capacity of $A$ s
\end{abstract}

\footnotetext{
(1) Parte da Tese de Doutorado do primeiro autor. Recebido para publicação em janeiro de 2005 e aprovado em julho de 2007.

(2) Professor do Departamento de Solos, Universidade do Estado de Santa Catarina - UDESC. Caixa Postal 281, CEP 88520-000 Lages (SC). E-mail: a2mlc@cav.udesc.br

(3) Professor do Departamento de Ciência do Solo, Universidade Federal de Lavras - UFLA. Caixa Postal 37, CEP 37200-000 Lavras (MG). Bolsista CNPq. E-mails: guilherm@ufla.br, niltcuri@ufla.br, jmarques@ufla.br e marx@ufla.br

(4) Engenheiro-Agrônomo, Departamento de Ciência do Solo, UFLA.

(5) Professora do Departamento de Ciência do Solo, UFLA. E-mail: lessi.antunes@bol.com.br
} 


\begin{abstract}
adsorption $\left(C M A D S_{A s}\right)$ in Oxisols. The total content was determined by the USEPA $3051 A$ method. The $\mathrm{cmADS} \mathrm{S}_{A s}$ was determined by the Langmuir Isotherms using six solution concentrations $\left(0,0.09,0.19,0.38,0.76,1.15 \mathrm{mmol} \mathrm{L}^{-1}\right)$ (1:100 soil: solution ratio), $p H$ values 5.5 and ionic strength $15 \mathrm{mmol} \mathrm{L} \mathrm{L}^{-1}$. In the 17 Oxisols the average total As content was $5.92 \mathrm{mg} \mathrm{kg}^{-1}$ and mean $\mathrm{cmADS}_{\mathrm{As}}$ was $2.013 \mathrm{mg} \mathrm{kg}^{-1}$. Clay, and $\mathrm{Fe}$ and $\mathrm{Al}$ oxides content influenced $\mathrm{cmADS}$ positively.
\end{abstract}

Index terms: sorption, chemical contamination, pollutants, trace elements.

\section{INTRODUÇÃO}

A manutenção da função agrícola e ecológica do solo passa preferencialmente pelo monitoramento de eventuais fontes de poluição. Estudos com elementostraço (Ets) em ecossistemas revelam que os solos têm sido contaminados com $\mathrm{Pb}, \mathrm{Cd}, \mathrm{Hg}$, As e outros (Alloway, 1990) e que o aumento das concentrações de Ets nos solos de áreas agrícolas resulta da deposição atmosférica e da aplicação de defensivos agrícolas, resíduos orgânicos e inorgânicos, fertilizantes e corretivos (Amaral Sobrinho et al., 1992). No caso específico do As, as adições antropogênicas ao solo advêm do uso de pesticidas e fertilizantes, de emissão derivada de mineração e fundição do ouro, chumbo, cobre e níquel, de produção de Fe e aço, da combustão de carvão e da irrigação com água contaminada (Sparks, 1995; Smith et al., 1999; Baird, 2002; Roychowdhury et al., 2002).

A ausência de regiões sem nenhuma entrada de contaminantes de origem antropogênica (Chen et al., 1999; Rice, 1999) possibilita a determinação apenas da concentração chamada de concentração de base (background), a qual é encontrada em um tempo particular durante um estudo ou programa de monitoramento. O conhecimento dessa concentração de As em Latossolos é de extrema relevância, já que estes constituem uma classe de solos que ocupa mais de $50 \%$ do território nacional (Oliveira, 2005).

Tão importante quanto o conhecimento da concentração de base de Ets é o conhecimento da capacidade de adsorção deles pelo solo. A capacidade de adsorção fornece indícios do poder-tampão do sistema, visto que muitos atributos do solo (como textura, teor de óxidos de $\mathrm{Fe}$ e $\mathrm{Al}$, entre outros) interferem na capacidade-tampão e, por conseqüência, influenciam a perda por lixiviação. A retenção seletiva de elementos-traço em sítios de troca na superfície de minerais de carga variável e nas arestas quebradas dos argilominerais tem sido identificada como adsorção específica ou adsorção química (formação de complexos de esfera interna) (McBride, 1994; Gustafsson \& Jacks, 1995; Smith et al., 1999); nos óxidos, ocorre ligação do tipo binuclear ou bidentada (Hiemstra \& van Riemsdijk, 1999).

A adsorção química do arsenato se dá principalmente em óxidos de $\mathrm{Fe}$ e $\mathrm{Al}$, aluminossilicatos amorfos e, em pequena extensão, em argilossilicatos (Smith et al., 1999). O arsenato é o ânion do ácido forte, $\mathrm{H}_{3} \mathrm{AsO}_{4}$, que possui os valores de pKa de 2,24, 6,94 e 11,5 (McBride, 1994). A espécie predominante de $\mathrm{As}(\mathrm{V})$ em soluções de solos ácidos é o $\mathrm{H}_{2} \mathrm{AsO}_{4}{ }^{-}$, enquanto na solução de solos alcalinos predomina a forma $\mathrm{HAsO}_{4}{ }^{2-}$ (Hayes \& Traina, 1998; Smith et al., 1998). As reações que controlam a disponibilidade de $\mathrm{As}(\mathrm{V})$ em solos compreendem adsorção/dessorção e precipitação/dissolução (Smith et al., 1998).

A adsorção de $\mathrm{As}(\mathrm{V})$ em diferentes adsorventes tem sido avaliada por isotermas de Freundlich e Langmuir e normalmente segue o modelo de cinética de primeira ordem, com constantes cinéticas de adsorção variáveis $\left(0,01\right.$ a $\left.0,14 \mathrm{~min}^{-1}\right)$ em função de temperatura, tipo de adsorvente e tamanho de partícula (Singh et al., 1996; Namasivayam \& Senthilkumar, 1998).

A alta toxicidade do As aos seres vivos e a possibilidade de existência de grande número de áreas contaminadas tornam de suma importância o entendimento dos processos de adsorção do As em solos tropicais, já que esses processos irão determinar a mobilidade e a biodisponibilidade do As no solo. Assim, este trabalho teve como objetivo conhecer os teores nativos e a capacidade máxima de adsorção de arsênio em Latossolos de diferentes regiões geográficas brasileiras.

\section{MATERIAL E MÉTODOS}

Foram utilizadas amostras da camada superficial $(0-20 \mathrm{~cm})$ de 15 Latossolos localizados em áreas experimentais das Embrapas e dois Latossolos do campus da UFLA, compondo assim um banco de dados representativo sobre Latossolos de todas as regiões geográficas brasileiras (Quadro 1). Esses Latossolos já foram alvo de outros estudos de adsorção (Guilherme et al., 1998; Pierangeli et al., 2001a,b,c, 2003, 2004; Campos et al., 2006), de teores semitotais de Ets (Campos et al., 2003), entre outros. A caracterização física, química e mineralógica desses solos pode ser encontrada em Silva et al. (1996) e Silva (1997).

Para determinação do teor de As, utilizou-se o protocolo do método USEPA 3051A (http:// www.epa.gov/SW-846/3051a.pdf), que prevê a digestão de 1,0 a $0,5 \mathrm{~g}$ de material em $10 \mathrm{~mL}$ de $\mathrm{HNO}_{3}$ concentrado em forno de microondas com tubos de Teflon® PTFE à pressão de $0,76 \mathrm{MPa}$ por $10 \mathrm{~min}$. As amostras de solo e de controle foram analisadas em 
Quadro 1. Classificação e localização dos solos estudados

\begin{tabular}{|c|c|c|c|}
\hline Solo & Classificação & Localização & Material de origem \\
\hline LVd 1 & Latossolo Vermelho distrófico típico & Passo Fundo - RS & Basalto e arenito (mistura) \\
\hline $\mathrm{LVd} 2$ & Latossolo Vermelho distrófico psamítico & Paranavaí - PR & Arenito Caiuá \\
\hline $\mathrm{LVd} 3$ & Latossolo Vermelho distrófico típico & Jaboticabal - SP & Basalto com influência de arenito \\
\hline $\mathrm{LVd} 4$ & Latossolo Vermelho distrófico típico & Lavras - MG & Gnaisse granítico mesocrático \\
\hline $\mathrm{LVd} 5$ & Latossolo Vermelho distrófico típico & Sete Lagoas - MG & Rochas pelíticas do grupo Bambuí \\
\hline $\mathrm{LVd} 6$ & Latossolo Vermelho distrófico típico & Goiânia - GO & Sedimentos argilosos retrabalhados \\
\hline $\mathrm{LVd} 7$ & Latossolo Vermelho distrófico típico & Planaltina - DF & Sedimento argilosos de cobertura \\
\hline $\mathrm{LVdf} 1$ & Latossolo Vermelho distroférrico típico & Ijuí - RS & Basalto \\
\hline LVdf 2 & Latossolo Vermelho distroférrico típico & Chapecó - SC & Basalto \\
\hline LVdf 3 & Latossolo Vermelho distroférrico típico & Londrina - PR & Basalto \\
\hline LVdf 4 & Latossolo Vermelho distroférrico típico & Dourados - MS & Rochas eruptivas básicas \\
\hline LVdf 5 & Latossolo Vermelho distroférrico típico & Ponta Grossa - PR & Rochas diversas do Pré-cambriano \\
\hline LVef 1 & Latossolo Vermelho eutroférrico típico & Campinas - SP & Diabásio \\
\hline LAd 1 & Latossolo Amarelo distrófico típico & Ubajara - CE & Arenito \\
\hline LAd 2 & Latossolo Amarelo distrófico típico & Lavras - MG & Gnaisse granítico leucocrático \\
\hline LAd 3 & Latossolo Amarelo distrófico típico & Areia - PB & Sedimento areno-argiloso \\
\hline LAx 1 & Latossolo Amarelo coeso típico & Tomé Açu - PA & Sedimentos do terciário \\
\hline
\end{tabular}

triplicata (Quadro 2). As amostras controle são utilizadas para comparar as baterias de digestão, facilitando assim a identificação de problemas durante esse processo.

Para determinação da capacidade máxima de adsorção, foram preparadas suspensões de TFSA dos 17 Latossolos em solução salina de $\mathrm{NaCl} 15 \mathrm{mmol} \mathrm{L}^{-1}$ com $\mathrm{pH}$ ajustado a 5,5 $( \pm 0,2)$. O ajuste do valor de $\mathrm{pH}$ foi realizado com $\mathrm{NaOH}$ e $\mathrm{HCl} 10 \mathrm{mmol} \mathrm{L}^{-1}$. A escolha do sal para preparo da solução de fundo (Quadro 3) se fundamenta na possibilidade de precipitação do arsenato na forma de arsenato de Ca, caso se optasse pela utilização de $\mathrm{CaCl}_{2} \mathrm{O}$ valor de pH 5,5 foi escolhido por dois motivos: por ser um dos valores de $\mathrm{pH}$ em que ocorre máximo de adsorção e por ser o mais próximo do valor médio de $\mathrm{pH}(4,8)$ dos Latossolos estudados.

As suspensões de cada solo foram colocadas para reagir com soluções de $\mathrm{Na}_{2} \mathrm{HAsO}_{4} \cdot 7 \mathrm{H}_{2} \mathrm{O}$ nas seguintes doses de As: 0, 90, 190, 380, 760 e $1.150 \mu \mathrm{mol} \mathrm{L}^{-1}$. Essas doses de As foram escolhidas após análise de especiação no programa MINTEQ (Quadro 3), no qual se observou que a maior dose de As que poderia ser utilizada sem que houvesse precipitação de sais de arsenato era de $1.500 \mu \mathrm{mol} \mathrm{L}{ }^{-1}$. A relação solo:solução final foi de 1:100, e todas as amostras foram avaliadas em triplicata.

O tempo de contato entre o solo e a solução foi de $72 \mathrm{~h}$, divididas em ciclos de $12 \mathrm{~h}$ de agitação e $12 \mathrm{~h}$ em repouso. Após as $72 \mathrm{~h}$ de reação, a suspensão de cada solo foi centrifugada e o sobrenadante retirado
Quadro 2. Teor de arsênio esperado e encontrado nas amostras controle e limite de detecção quantitativo (LMQ)

\begin{tabular}{clc}
\hline \multirow{2}{*}{ Amostra controle } & \multicolumn{2}{c}{ Teor } \\
\cline { 2 - 3 } & Esperado & Encontrado \\
\cline { 2 - 3 } & \multicolumn{2}{c}{$\mathrm{mg} \mathrm{kg}^{-1}$} \\
\hline AC $02-115$ & $1,86 \pm 0,2$ & $1,44 \pm 0,03$ \\
AC $02-103$ & $3,75 \pm 1,6$ & $2,09 \pm 0,51$ \\
ESAL 1 & - & $9,73 \pm 4,54$ \\
LDQ & 0,013 & \\
\hline
\end{tabular}

(1) $L D Q=F d^{*}\left(M \pm k^{*} \mathrm{~s}\right)$ (ALPHA, 1989), em que $L D Q$ : limite de detecção qualitativo do método analítico; $F d$ : fator de diluição das amostras; $M$ : média das provas em branco; $k$ : coeficiente de Kaiser que possui valor fixo igual a 3 (Skoog, 2002); e $s$ : desvio-padrão das provas em branco.

para leitura. A quantificação do teor de As em todas as análises foi realizada por espectrofotometria de absorção atômica, usando equipamento Perkin Elmer Aanalyst 800, com atomização eletrotérmica em forno de grafite. As condições de leitura utilizadas foram temperatura de pirólise de $500{ }^{\circ} \mathrm{C}$ (recomendação do fabricante) e $0,003 \mathrm{mg}$ de $\mathrm{Mg}$ na forma de $\mathrm{Mg}\left(\mathrm{NO}_{3}\right)_{2}$ como modificador químico (Niedzielski et al., 2002).

Para obtenção da $\mathrm{CMADS}_{\mathrm{As}}$ foram estabelecidas as isotermas de adsorção, mais precisamente, 
isotermas de Langmuir, sendo estas as mais utilizadas no estudo de adsorção de As (Singh et al., 1996; Namasivayam \& Senthilkumar, 1998; Ladeira et al., 2002). A equação da isoterma de Langmuir, $\frac{\mathrm{x}}{\mathrm{m}}=\frac{\mathrm{Ce}}{\frac{1}{\mathrm{k}}+\mathrm{Ce}}$, apresenta x como a quantidade de As adsorvido; m, a quantidade do adsorvente; Ce, a concentração de equilíbrio da solução; e k, a constante de ajuste. Em altas concentrações do adsorvato, a quantidade adsorvida se torna constante, ou seja, a superfície do adsorvente torna-se saturada e alcança um máximo de adsorção ou CMADS do elemento estudado (Tan, 1992). Para avaliar a correlação entre algumas características químicas, físicas e mineralógicas e a $\mathrm{CMAD}_{\mathrm{AS}}$, foi efetuada análise de correção entre eles (Quadro 4).

Quadro 3. Dados referentes à especiação conduzida no programa MINTEq para maior dose de As $\left(1,5 \mathrm{mmol} \mathrm{L}^{-1}\right)$ utilizada no experimento CMADS $_{\text {As }}$. Na simulação foram considerados NaCl 15 mmol L m $^{-1}$ pH 5,5

\begin{tabular}{|c|c|c|}
\hline Componente & $\begin{array}{l}\text { Concentração total } \\
\text { do componente }\end{array}$ & Espécie \\
\hline \multicolumn{3}{|c|}{$\%$} \\
\hline \multirow[t]{2}{*}{$\mathrm{Cl}^{-}$} & 99,424 & $\mathrm{Cl}^{-}$ \\
\hline & 0,576 & $\mathrm{NaCl}(\mathrm{aq})$ \\
\hline \multirow[t]{3}{*}{$\mathrm{AsO}_{4}{ }^{3-}$} & 4,810 & $\mathrm{HAsO}_{4}{ }^{2-}$ \\
\hline & 95,144 & $\mathrm{H}_{2} \mathrm{AsO}_{4}^{-}$ \\
\hline & 0,046 & $\mathrm{H}_{3} \mathrm{AsO}_{4}$ \\
\hline \multirow[t]{2}{*}{$\mathrm{Na}^{+}$} & 99,424 & $\mathrm{Na}^{+}$ \\
\hline & 0,576 & $\mathrm{NaCl}(\mathrm{aq})$ \\
\hline
\end{tabular}

\section{RESULTADOS E DISCUSSÃO}

Os Latossolos avaliados no presente trabalho apresentam teor médio de As de $5,2 \mathrm{mg} \mathrm{kg}^{-1}$ (Figura 1). Dos 17 solos, 16 apresentaram teor de As abaixo de $10 \mathrm{mg} \mathrm{kg}^{-1}$, valor este apresentado por Fitz \& Wenzel (2002) e Smedley \& Kinniburgh (2002) para solos não-contaminados.

A exceção é o Latossolo Vermelho distroférrico típico (LVdf 5), que apresentou 31,7 $\pm 12,6 \mathrm{mg} \mathrm{kg}^{-1}$ de As no solo. O teor de As de LVdf 5 pode ser considerado normal, quando comparado com o intervalo de valor para solos não-contaminados apresentado no boletim da WHO (2001): 1-40 mg kg-1. O teor de As do LVdf 5 também se encontra abaixo do observado em Latossolo Ferrífero (36 mg kg-1 de As) por Curi \& Franzmeier (1987) e em Latossolos sob vegetação do Cerrado (38 $\mathrm{mg} \mathrm{kg}^{-1}$ de As) por Marques (2000). Os teores apresentados na figura 1 , mesmo sendo considerados

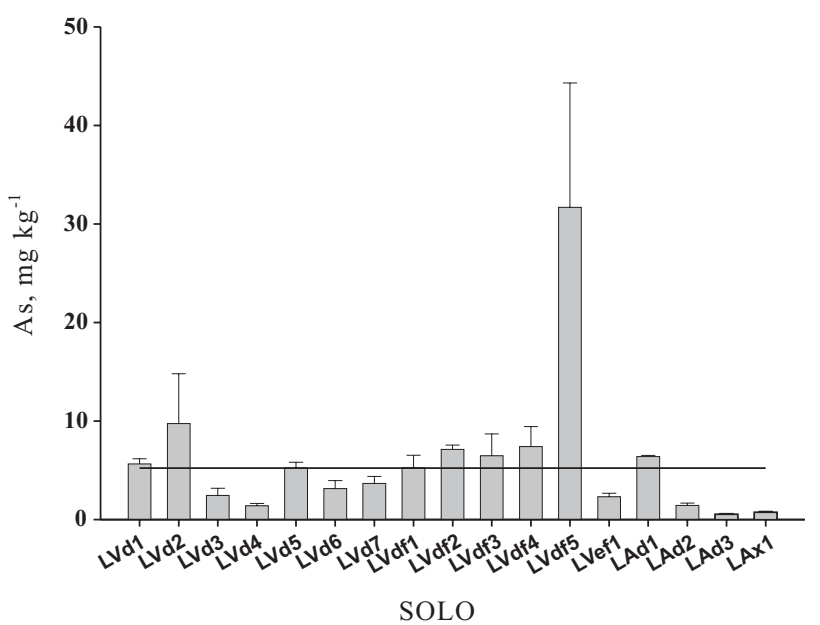

Figura 1. Teor semitotal de As nos 17 Latossolos brasileiros determinado após digestão pelo método USEPA 3051A. As barras acima das colunas indicam o desvio-padrão, e a linha, o teor médio de As dos Latossolos.

semitotais, podem ser utilizados como valores de referência para solos não contaminados, pois representam o máximo do As disponível.

As equações de regressão linear, usadas para cálculo da CMADS, foram significativas a $5 \%$ (Figura 2), e o coeficiente de correlação entre o valor predito e o observado variou de 0,56 a 0,99.

A CMADS $_{\mathrm{As}}$ média para os 17 Latossolos foi de $2.012 \mathrm{mg} \mathrm{kg}^{-1}$ (Figura 3); o LAx apresentou a menor (753 $\mathrm{mg} \mathrm{kg}^{-1}$ de As) e o LVdf 4 a maior capacidade de adsorção (3.449 mg kg-1). A capacidade máxima de adsorção de LVdf 4 assemelha-se à encontrada para Latossolo coletado em Paracatu-MG, de 3.200 mg kg-1 (Ladeira \& Ciminelli, 2004). A capacidade de adsorção dos solos estudados é superior à encontrada para 16 solos chineses, que variou de 21,9 a 524,86 mg kg-1 (Jiang et al., 2005a,b). Essa menor capacidade de adsorção dos solos chineses deve estar relacionada ao baixo teor de argila $\left(<460 \mathrm{~g} \mathrm{~kg}^{-1}\right)$, óxido de $\mathrm{Fe}\left(<5 \mathrm{~g} \mathrm{~kg}^{-1}\right)$ e óxido de alumínio $\left(<2 \mathrm{~g} \mathrm{~kg}^{-1}\right)$. A alta capacidade de adsorção observada no solo LVdf 4, o qual apresenta os maiores teores de argila, está de acordo com a afirmação de que o teor de argila e os componentes minerais dessa fração controlam a adsorção de oxiânions como o arsenato (Jacobs et al., 1970; Smith et al., 1999). É conveniente salientar que, em sistemas complexos como o solo, características como textura, natureza dos constituintes minerais, $\mathrm{pH}$ e presença de íons competidores (Smith et al., 1999) determinaram uma particular $\mathrm{CMADS}_{\mathrm{As}}$ para cada solo estudado.

A CMADS $_{\mathrm{As}}$ dos 17 Latossolos apresentou correlação significativa positiva com o $\mathrm{Fe}_{2} \mathrm{O}_{3}, \mathrm{Fe}_{\mathrm{DCB}}$, $\mathrm{Al}_{2} \mathrm{O}_{3}, \mathrm{SiO}_{2}$, superfície específica (SE), hematita e teor de argila (Quadro 4). A correlação positiva com óxidos era esperada, já que, à semelhança do fosfato, o arsenato é altamente adsorvido a óxidos, especialmente 


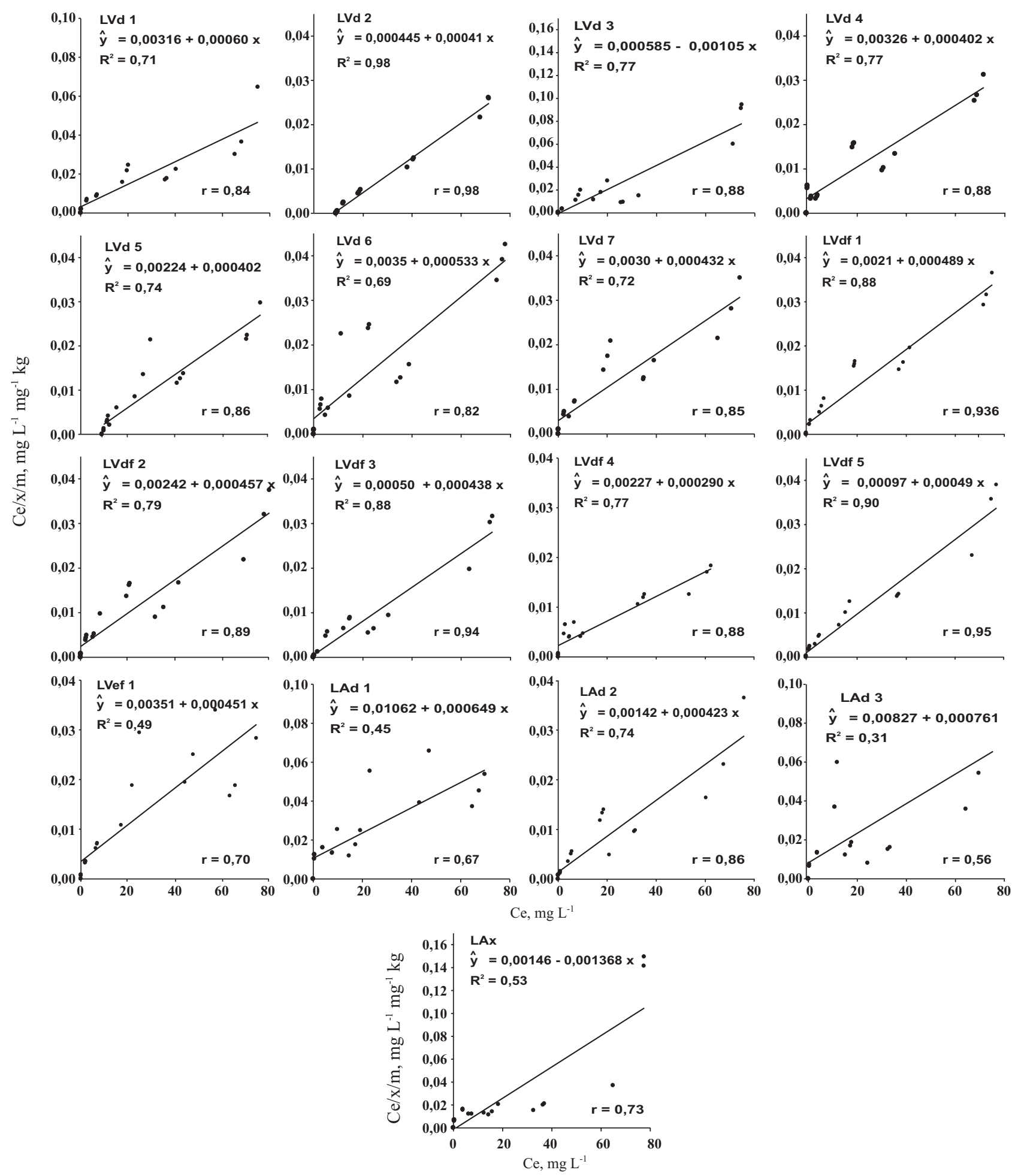

Figura 2. Regressões lineares utilizadas no cálculo da capacidade máxima de adsorção de As $\left(\mathrm{CMADS}_{\mathrm{As}}\right)$ das amostras do horizonte A dos 17 Latossolos avaliados.

em condições de solos ácidos (McBride, 1994). Ladeira et al. (2002) observaram que os solos com elevado teor de óxidos de $\mathrm{Fe}(11 \%)$ e $\mathrm{Al}(26 \%)$ e alta área superficial específica $\left(35,7 \mathrm{~m}^{2} \mathrm{~g}^{-1}\right)$ apresentaram maior capacidade de adsorção de As. O índice $\mathrm{Kr}$ mostrou correlação significativa negativa com a $\mathrm{CMADS}_{\mathrm{As}}$, ou seja, entre os Latossolos avaliados neste estudo existe tendência de aqueles com menor índice Kr (LVd 2, LVd 4, LVdf 4, LVdf 5, LVef 1 e LAd 2) adsorverem mais arsenato. 


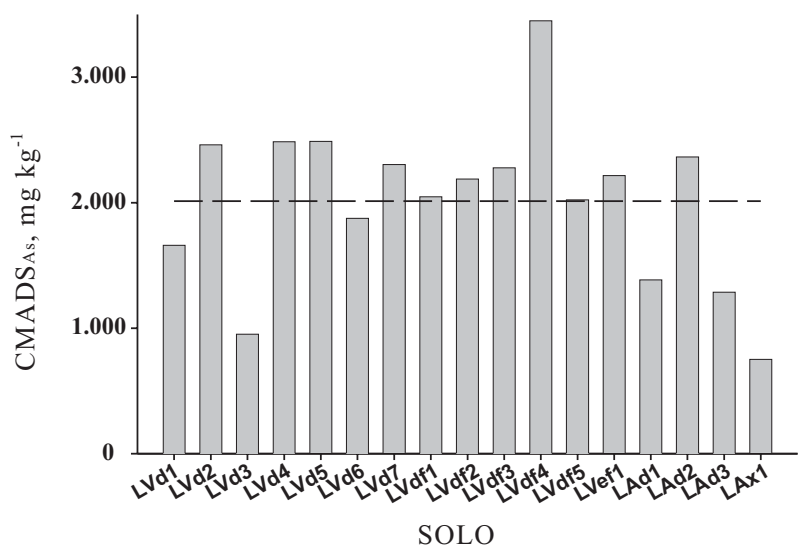

Figura 3. Capacidade máxima de adsorção de As das amostras do horizonte A dos 17 Latossolos. A CMADS $_{\mathrm{As}}$ foi obtida pela equação: $\mathrm{CMADS}_{\mathrm{As}}=$ $1 / b$, sendo $b$ a inclinação da reta obtida por regressão linear (Figura 2).

Quadro 4. Coeficiente de correlação entre os atributos físicos, químicos e mineralógicos de 17 Latossolos brasileiros e $\mathrm{CMADS}_{\mathrm{As}}$, a qual foi obtida pela equação: $\operatorname{CMADS}_{\mathrm{As}}=1 / \mathrm{b}$, sendo $\mathrm{b}$ a inclinação da reta

\begin{tabular}{lc}
\hline \multicolumn{1}{c}{ Atributo } & $\begin{array}{c}\text { Correlação (r) } \\
\text { CMAD }_{\mathrm{As}}\end{array}$ \\
\hline Argila & $0,46^{*}$ \\
$\mathrm{MO}$ & $-0,09^{\mathrm{ns}}$ \\
$\mathrm{SE}$ & $0,43^{*}$ \\
$\mathrm{Fe}_{2} \mathrm{O}_{3}$ (ataque sulfúrico) & $0,43^{*}$ \\
$\mathrm{Al}_{2} \mathrm{O}_{3(\text { ataque sulfúrico) }}$ & $0,63^{* *}$ \\
$\mathrm{SiO}_{2 \text { (ataque sulfúrico) }}$ & $0,42^{*}$ \\
$\mathrm{Fe}_{\text {DCB }}$ & $0,52^{*}$ \\
$\mathrm{Feox}$ & $0,37^{\mathrm{ns}}$ \\
$\mathrm{Ki}_{\mathrm{Kr}}$ & $0,02^{\mathrm{ns}}$ \\
Caulinita & $-0,42^{*}$ \\
Gibbsita & $0,19^{\mathrm{ns}}$ \\
$\mathrm{Hematita}$ & $0,27^{\mathrm{ns}}$ \\
Goethita & $0,51^{*}$ \\
\hline
\end{tabular}

${ }^{\text {ns }}$ e *: Não-significativo e significativo a $5 \%$.

\section{CONCLUSÃO}

1. Os teores médios de As dos 17 Latossolos apresentaram-se dentro da faixa de teores normalmente encontrada em solos ditos não-contaminados. Esses teores médios de As podem ser utilizados como inferências para essa classe de solos e no monitoramento de eventuais contaminações.

2. $\mathrm{ACMADS}_{\mathrm{As}}$ apresentou correlação positiva com o teor de argila, com a área superficial específica e com o teor e tipo de óxidos de $\mathrm{Fe}$ e $\mathrm{Al}$ presentes na fração argila.

3. Solos com menor índice $\mathrm{Kr}$ apresentaram maior capacidade de adsorver arsênio.

\section{LITERATURA CITADA}

ALLOWAY, B.J. Heavy metals in soil. New York, John Wiley \& Sons, 1990. 339p.

AMARAL SOBRINHO, N.M.B.; COSTA, L.M.; OLIVEIRA, C. \& VELLOSO, A.C.X. Metais pesados em alguns fertilizantes e corretivos. R. Bras. Ci. Solo, 16:271-276, 1992.

BAIRD, C. Química ambiental. 2.ed. Porto Alegre, Bookman, 2002. 621p.

CAMPOS, M.L.; GUILHERME, L.R.G.; VISIOLLI, E.; ANTUNES, A.S.; CURI, N.; MARQUES, J.J. \& SILVA, M.L.N. Força iônica da solução de equilíbrio na adsorção de arsênio em Latossolos brasileiros. Pesq. Agropec. Bras., 4:457-460, 2006.

CAMPOS, M.L.; PIERANGELI, M.A.P.; GUILHERME, L.R.G.; MARQUES, J.J. \& CURI, N. Baseline concentration of heavy metals in Brazilian Latosols. Comm. Soil Sci. Plant Anal., 34:547-557, 2003.

CHEN, M.; MA, L.Q. \& HARRIS, W.G. Baseline concentrations 15 trace elements in Florida surface soils. J. Environ. Qual., 28:1173-1181, 1999.

CURI, N. \& FRANZMEIER, D.P. Effect of parent rocks on chemical and mineraligical properties of some Oxisols in Brasil. Soil Sci. Soc. Am. J., 51:153-158, 1987.

FITZ, W.J. \& WENZEL, W.W. Arsenic transformations in soil-rhizosphere-plant system: fundamentals and potential aplication to phytoremediation. J. Biotechnol., 99:259-278, 2002.

GUILHERME, L.R.G. \& ANDERSON, S.J. Copper sorption kinetics in two oxide-rich soils (Oxisols): Effect of phosphate pretreatment. In: JENNE, E.A., ed. Adsorption of metals by geomedia: Variables, mechanisms, and model applications. San Diego, Academic Press, 1998. p.209228.

GUSTAFSSON, J.P. \& JACKS, G. Arsenic geochemistry in forested soil profiles as revealed by solid-phase studies. Appl. Geochem., 10:307-315, 1995. 
HAYES, K.F. \& TRAINA, S.J. Metal speciation and its significance in ecosystem health. In: HUANG, P.M., ed. Soil chemistry and ecosystem health. Soil Sci. Soc. Am. J., 1998. p.45-84. (SSSA Special Publication, 52)

HIEMSTRA T. \& van RIEMSDIJK, W.H. Surface structural ion adsorption modeling of competitive binding of oxyanions by metal (hydr)oxides. J. Colloid Inter. Sci., 210:182-193, 1999.

JACOBS, L.W.; SYERS, J.K. \& KEENEY, D.R. Arsenic sorption by soils. Soil Sci. Soc. Am. Proc., 34:750-754, 1970.

JIANG, W.; ZHANG, S.; SHAN, X.; FENG, M.; ZHU, Y.; MCLAREN, R.G. Adsorption of arsenate on soils. Part 2: Modelingthe relationship between adsorption capacity and soil physiochemical properties using 16 Chinese soils. Environ. Poll., 138:285-289, 2005a.

JIANG, W.; ZHANG, S.; SHAN, X.; FENG, M.; ZHU, Y. \& MCLAREN, R.G. Adsorption of arsenate on soils. Part 1: Laboratory batch experiments using 16 Chinese soils with different physiochemical properties. Environ. Poll., 138:278-284, 2005b.

KABATA-PENDIAS, A. \& PENDIAS, H. Trace elements in soil and plant. 3.ed. Boca Raton, CRC, 2001. 413p.

LADEIRA, A.C.Q. \& CIMINELLI, V.S.T. Adsorption and desorption of arsenic on an oxisol and its constituents. Water Res., 38:2087-2094, 2004

LADEIRA, A.C.Q.; CIMINELLI, V.S.T. \& NEPOMUCENO, A.L. Seleção de solos para imobilização de arsênio. R. Esc. Minas, 55:215-221, 2002

MARQUES, J.J.G.S.M. Trace element distributions in Brazilian Cerrado at the landscape and micrometer scales. West Lafayette, Purdue University, 2000. 173p. (Tese de Doutorado)

MCBRIDE, M.B. Environmental chemistry of soils. New York, Oxford University, 1994. 406p.

NAMASIVAYAM, C \& SENTHILKUMAR, S. Removal of arsenic (V) from aqueos solution using industrial solid waste: Adsorption rates and equilibrium studies. Ind. Eng. Chem. Res., 37:4816-4822, 1998.

NIEDZIELSKI, P.; SIEPAK, M. \& SIEPAK, J. Comparison of modifiers for determination of arsenic, antimony and selenium by atomic absorption spectrometry with atomization in graphite tube or hydride generation and in-situ preconcentration in graphite tube. Microchem. J., 72:137-1445, 2002.

OLIVEIRA, J.B. Pedologia aplicada. Piracicaba, FEALQ, 2005. $574 \mathrm{p}$.

PIERANGELI, M.A.P.; GUILHERME, L.R.G.; CURI, N.; ANDERSON, S.J. \& LIMA, J.M. Adsorção e dessorção de cádmio, cobre e chumbo por amostras de Latossolos prétratadas com fósforo. R. Bras. Ci. Solo, 28:377-384, 2004.
PIERANGELI, M.A.P.; GUILHERME, L.R.G.; CURI, N.; SILVA, M.L.N.; LIMA, J.M. \& COSTA, E.T.S. Efeito do pH na adsorção e dessorção de cádmio em Latossolos brasileiros. R. Bras. Ci. Solo, 29:523-532, 2005.

PIERANGELI, M.A.P.; GUILHERME, L.R.G.; CURI, N.; SILVA, M.L.N.; OLIVEIRA, L.R. \& LIMA, J.M. Efeito do $\mathrm{pH}$ na adsorção-dessorção de chumbo em Latossolos brasileiros. R. Bras. Ci. Solo, 25:269-277, 2001a.

PIERANGELI, M.A.P.; GUILHERME, L.R.G.; CURI, N.; SILVA, M.L.N.; OLIVEIRA, L.R. \& LIMA, J.M. Teor total e capacidade máxima de adsorção de chumbo em Latossolos brasileiros. R. Bras. Ci. Solo, 25:279-288, 2001b.

PIERANGELI, M.A.P.; GUILHERME, L.R.G.; OLIVEIRA, L.R.; CURI, N. \& SILVA, M.L.N. Efeito da força iônica da solução de equilíbrio na adsorção de cádmio em Latossolos brasileiros. Pesq. Agropec. Bras., 38:737-745, 2003.

PIERANGELI, M.A.P.; GUILHERME, L.R.G.; OLIVEIRA, L.R.; CURI, N. \& SILVA, M.L.N. Efeito da força iônica da solução de equilíbrio sobre a adsorção-dessorção de chumbo em Latossolos brasileiros. Pesq. Agropec. Bras., 36:1077-1084, 2001c.

RICE, K.C. Trace-element concentrations in streambed sediment across the conterminous United States. Environ. Sci. Technol., 33:2499-2504, 1999.

ROYCHOWDHURY, T.; UCHINO, T.; TOKUNAGA, H. \& ANDO, M. Arsenic and other heavy metals in soils from an arsenic-affected area of West Bengal, India. Chemosphere, 49:605-618, 2002.

SILVA, M.L.N. Erosividade da chuva e proposição de modelos para estimar a erodibilidade de Latossolos brasileiros. Lavras, MG, Universidade Federal de Lavras, 1997. 154p. (Tese de Doutorado)

SILVA, M.L.N.; CURI, N.; MARQUES, J.; GUILHERME, L.R.G. \& LIMA, J.M. Ponto de efeito salino nulo e suas relações com propriedades mineralógicas e químicas de Latossolos brasileiros. Pesq. Agropec. Bras., 31:663-671, 1996.

SINGH, D.B.; PRASAD, G. \& RUPAINWAR, D.C. Adsorption technique for the treatment of As (V)-rich effluents. Colloids Surfaces A: Physicochem. Eng. Aspects, 111:4956, 1996.

SKOOG, D.A. Princípios de análise instrumental. 5.ed. Porto Alegre, Bookman, 2002. 836p.

SMEDLEY, P.L. \& KINNIBURGH, D.G. A review of the source, behaviour and distribution of arsenic in natural waters. Appl. Geochem., 17:517-568, 2002.

SMITH, E.; NAIDU, R. \& ALSTON, A.M. Arsenic in the soil environment: a review. Adv. Agron., 64:149-195, 1998.

SMITH, E.; NAIDU, R. \& ALSTON, A.M. Chemistry of arsenic in soils: I. Sorption of arsenate and arsenite by four Australian soils. J. Environ. Qual., 28:1719-1726, 1999.

SPARKS, D.L. Environmental soil chemistry. San Diego, Academic, 1995. 265p. 
TAN, K.H. Principles of soil chemistry. 2.ed. New York, Marcel Dekker, 1992. 362p.

USEPA. Test methods for evaluating solid waste, physical/ chemical methods. SW-846. 3.ed. Washington, U.S. Gov. Print. Office , 1995.
WHO. Arsenic and arsenic compounds. 2.ed. 2001. 187 p. (Environmental Health Criteria Series, 224). Disponível em: <http://www.inchem.org/documents/ehc/ehc/ ehc224.htm>. Acesso Julho de 2005. 